\title{
Imaging the past: Neural activation in frontal and temporal regions during regular and irregular past-tense processing
}

\author{
MARC F. JOANISSE \\ University of Western Ontario, London, Ontario, Canada \\ and \\ MARK S. SEIDENBERG \\ University of Wisconsin, Madison, Wisconsin
}

\begin{abstract}
This article presents fMRI evidence bearing on dual-mechanism versus connectionist theories of inflectional morphology. Ten participants were scanned at 4 Tesla as they covertly generated the past tenses of real and nonce (nonword) verbs presented auditorily. Regular past tenses (e.g., walked, wugged) and irregular past tenses (e.g., took, slept) produced similar patterns of activation in the posterior temporal lobe in both hemispheres. In contrast, there was greater activation in left and right inferior frontal gyrus for regular past tenses than for irregular past tenses. Similar previous results have been taken as evidence for the dual-mechanism theory of the past tense (Pinker \& Ullman, 2002). However, additional analyses indicated that irregulars that were phonologically similar to regulars (e.g., slept, fled, sold) produced the same level of activation as did regulars, and significantly more activation than did irregulars that were not phonologically similar to regulars (e.g., took, gave). Thus, activation patterns were predicted by phonological characteristics of the past tense rather than by the rule-governed versus exception distinction that is central to the dual-mechanism framework. The results are consistent with a constraint satisfaction model in which phonological, semantic, and other probabilistic constraints jointly determine the past tense, with different degrees of involvement for different verbs.
\end{abstract}

Language is often construed in terms of a mental grammar incorporating symbolic rules, of which the English past tense (bake-baked, walk-walked) is a well-studied example (Bybee \& Slobin, 1982; Halle \& Mohanan, 1985; Pinker, 1999). However, in English, as in many other languages, there are irregular forms that violate the rules (e.g., took, gave). Several theories have addressed how rule-governed forms and exceptions are learned, represented, and used. Traditional linguistic approaches make a strong distinction between rules of grammar and the lexicon. Thus, regular forms such as walked are thought to be generated by a rule, with exceptions such as took stored in and retrieved from a mental dictionary or lexicon. This account reflects a long-standing view within linguistic theory that the lexicon includes all and only in-

This research was supported by grants from the Canadian Institutes for Health Research, the Natural Sciences and Engineering Research Council of Canada, and the Canada Foundation for Innovation to M.F.J., and by National Institute of Mental Health (U.S.) Grants KO2 MH 01188 and RO1 MH58723 to M.S.S. We thank J. Gati and C. Thomas for assistance with fMRI imaging and analyses and the Robarts Research Institute (London, ON) for fMRI services. We are grateful to Jason Zevin, Matthew Lambon Ralph, Karalyn Patterson, and James McClelland for their useful comments on this work. Correspondence should be addressed to M. F. Joanisse, Department of Psychology, University of Western Ontario, London, ON, N6A 5C2 Canada (e-mail: marcj@uwo.ca). formation that cannot be derived by more general grammatical mechanisms (Spencer, 1991).

In 1986, Rumelhart and McClelland proposed an alternative view: that all past tenses are generated by the same mechanism, a connectionist network. There were three principal motivations for this approach. First, they noted that most irregular past tenses are not arbitrary; rather, they tend to show many partial regularities. As is widely noted, there are pools of phonologically similar irregular past tenses, such as ring-rang, sing-sang, springsprang or sleep-slept, keep-kept, weep-wept (Halle \& Mohanan, 1985; Pinker, 1991). These reflect subpatterns within the putatively irregular forms. However, there are also similarities between many regular and irregular pasttense forms. For example, the regular past tense (e.g., bake-baked) retains the onset and coda of the present tense (/b/ and / $/$, respectively). The same occurs in forming irregular past tenses such as took, sat, and ran, which retain the onsets and codas of take, sit, and run, respectively. Similarly, the codas of irregular past tenses such as hit, said, and fled are the same as regular past-tense endings (e.g., baked, baled). The partial systematicity of irregulars is not captured by systems that treat fully regular forms and exceptions as unrelated. The second motivation for this approach was that connectionist networks employing distributed representations can learn and represent both fully regular forms and exceptions - and, im- 
portantly, the differing degrees of overlap between the two (for early illustrations of this point, see Daugherty \& Seidenberg, 1992; MacWhinney \& Leinbach, 1991). This approach contradicted the claim that regular and irregular forms require separate mechanisms (Pinker, 1991). Finally, Rumelhart and McClelland's approach offered an alternative account of generalization: Rather than involving rules, generalization is achieved by passing novel patterns through a network trained via exposure to familiar forms.

In response to Rumelhart and McClelland's (1986) work, Pinker and colleagues developed a hybrid model consisting of the standard grammatical rule mechanism but also an "associative net" to handle the exceptions (Pinker, 1991; Ullman et al., 1997). The latter mechanism replaced the list of exceptions in traditional theories and was intended to capture the nonarbitrary phonological relations among irregular forms such as sing-sang and ring-rang. This theory nevertheless maintained the idea that symbolic rules are used for generating regular past tenses and generalizing the -ed form to novel forms that occasionally enter the vocabulary (e.g.,faxed). Although the theory has undergone revisions and extensions over the years (see Pinker, 1999; Ullman, 2001), it continues to emphasize the isolability of the two proposed subsystems: They are thought to be distinct modules that process different types of words (rule governed vs. exception), are governed by different computational principles (application of symbolic rules vs. activation of associative memory), and are acquired by different mechanisms (rule induction vs. rote learning). A large body of research has been taken as supporting this theory, including evidence concerning past-tense acquisition in children (Marcus et al., 1992), normal adult processing (Prasada \& Pinker, 1993), cross-linguistic studies (Marcus, Brinkmann, Clahsen, Wiese, \& Pinker, 1995), and breakdown in aphasic patients (Ullman et al., 1997). Thus, the research provides broad evidence that symbolic rules are an important component of language and for a specific theory of the past tense and other aspects of inflectional morphology.

This research has been highly controversial, however, with many responses to the work focusing on issues such as the necessity of symbolic rules, the adequacy of connectionist models, the characterization and interpretation of behavioral data, the lack of computational specificity in the dual-mechanism approach, and so on (Daugherty \& Seidenberg, 1992; Haskell, MacDonald, \& Seidenberg, 2003; MacWhinney \& Leinbach, 1991; Plunkett \& Marchman, 1993; Ramscar, 2002). Perhaps the main problem concerns the claim that because the regular and irregular forms are governed by distinct subsystems, they "should be dissociable from virtually every point of view" (Pinker, 1991, p. 532). Whether these systems actually behave as differently as claimed has been repeatedly questioned. To take one representative example, generalization (forming the past tense of a novel form such as wug) is said to be the purview of the rule module, and indeed provides some classic evidence that lan- guage involves symbolic rules. However, generation of novel past tenses is affected by lexical knowledge (e.g., the past tense of spling is affected by phonological neighbors such as sing and ring, yielding splang as the past tense instead of splinged), as well as by semantic information (e.g., people say that the past tense of frink is frank in a context that emphasizes eating and drinking but frinked in other contexts; Ramscar, 2002). Thus, the original strong claim (Pinker \& Prince, 1988) that generalization is accomplished by a rule module that applies blindly to any string not listed in the lexicon without reference to phonological or semantic factors has been weakened over the years in the face of counterevidence. For instance, in response to the finding that generating past tenses of novel words is affected by similarity to existing irregulars (e.g., spling-splang, instead of splinged), it has been suggested that "the rule could fail to apply to a new word if the word is so similar to irregular words in memory that analogy is irresistible" (Pinker, 1999). The mechanism by which nonwords are actually drawn into the associative net responsible for exceptions has not been specified, however, and so it is not clear whether this account makes correct predictions about which items are affected. Moreover, the "analogy" method, if spelled out explicitly, could apply as well to fully regular forms, obviating the need for a rule entirely. Similar issues have arisen with respect to other types of data initially argued to support the dual-mechanism view (see, e.g., Haskell et al., 2003; McClelland \& Patterson, 2002).

A variety of connectionist models of inflectional morphology have been proposed over the years, focusing on different aspects of the phenomena (e.g., acquisition, skilled performance, cross-linguistic differences, effects of brain injury). Building on Rumelhart and McClelland's (1986) original proposal, we developed a general theoretical framework in which the production of morphological forms is seen as a constraint satisfaction problem, with the correct form determined by multiple probabilistic sources of linguistic information (Joanisse \& Seidenberg, 1999). These constraints principally involve semantic and phonological information but may include other factors such as orthographic knowledge if the task involves reading, pragmatic considerations (e.g., avoiding ambiguity; Haskell et al., 2003), and context if one is provided (Ramscar, 2002). We have focused primarily on phonology and semantics, which are sufficient to account for many facts about skilled performance.

Our model, illustrated in Figure 1, differs from previous models, dating from the original Rumelhart and McClelland (1986) work, which have focused on the classic task of transforming a phonological representation of the present tense into a phonological past tense (Berko, 1958). As Pinker and Prince (1988) noted, such models have limited scope; for example, they cannot distinguish homophones such as right and write, which are associated with both regular and irregular past tenses (righted and wrote, respectively). They also noted that changing the model so that it instead mapped from semantics to phonology would not solve the problem because of verbs such as slap, 


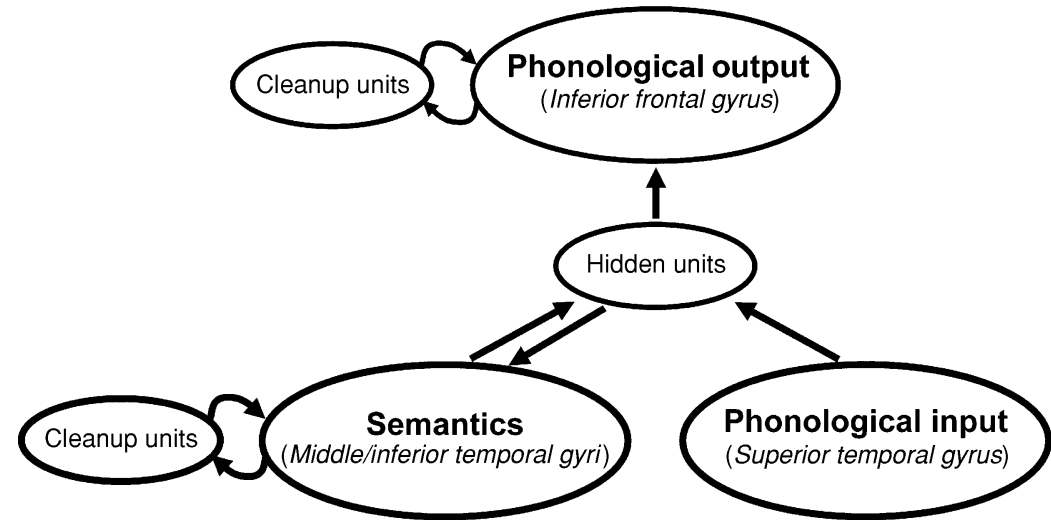

Figure 1. Architecture of the Joanisse and Seidenberg (1999) model of morphology. The model represents information about a word's sound (phonological input and phonological output) and meaning (semantics). Past-tense forms are computed as the convergence of these codes. Ellipses represent groups of artificial neurons, and arrows indicate directional weighted connections between groups. The network learns relationships among words' phonological and semantic forms through the adjustment of these connection weights as a function of experience. Cleanup and hidden units represent groups of neurons that do not receive external input, but which provide the network with additional computational and representational capacity. Brain regions that are suggested to be associated with these knowledge types are indicated in parentheses.

strike, and hit, which are semantically similar but form their past tenses differently. Pinker and Prince therefore concluded that connectionist models could not account for facts about the past tense. Marcus et al. (1995) have made the same argument with reference to plural formation in German.

These arguments ignore a fundamental property of connectionist networks, which can exploit multiple simultaneous constraints. Thus, in the Joanisse and Seidenberg (1999) model, past tenses are determined by both semantic and phonological constraints, with different forms relying on the constraints in different degrees, as determined through learning. For example, the semantic differences between right and write, rather than their sounds, differentiate between them and thus determine whether the past tense is righted or wrote. Both semantic and phonological codes work together; for homophones such as /rayt/, semantics is decisive. Conversely, there are greater phonological differences than semantic ones among slap, strike, and hit, and so phonology exerts a greater influence in determining their past tenses. In both cases, the conjunction of semantic and phonological information unambiguously determines the correct past tense; what differs is simply the degree to which the different types of information are decisive for different verbs. The balance between these probabilistic sources of information is learned through exposure to examples. (For another illustration, see Harm \& Seidenberg, 2004, who applied the same idea to the more complex problem of determining the meaning of a word from a conjunction of orthographic and phonological constraints.) Thus, how well a past tense is formed is a graded function of the extent to which it satisfies the constraints in the network used to generate all forms. ${ }^{1}$
At this point, then, two competing accounts are under consideration, one closely tied to the notion of symbolic rules and the other to connectionist networks that rapidly and efficiently exploit simultaneous probabilistic constraints. Much of the behavioral data can be explained at least in broad terms by both theories, although the connectionist account has been worked out at a greater level of computational specificity. Thus, the debate about the proper treatment of the past tense has continued without resolution. The controversy continues to be of major interest to cognitive scientists and neuroscientists because it involves different views about the nature of language and other higher cognitive capacities. The resolution of this debate is thus likely to have implications for the study of other issues as well.

\section{Neuroimaging Evidence}

In recent years, the past-tense debate has come to focus on evidence concerning the brain bases of this knowledge, particularly neuroimaging data. Does the brain respect a strong distinction between rule-governed forms and irregular forms, as dual-mechanism theories suggest? Or is the production of the past tense (and other inflected forms) determined by semantic, phonological, and other constraints, represented in brain areas independently known to code these types of information? Several neuroimaging studies have addressed these issues. The standard methodology has been to examine whether regulars and irregulars produce different patterns of activation, using positron emission tomography (PET; Jaeger et al., 1996), functional magnetic resonance imaging (fMRI; Beretta et al., 2003), ${ }^{2}$ event-related potentials (ERP; Lavric, Pizzagalli, Forstmeier, \& Rippon, 2001), or magnetoencephalography (MEG; Dhond, Marinkovic, 
Dale, Witzel, \& Halgren, 2003). The basic logic of these studies was that differences in brain activity associated with regulars and irregulars would indicate that they are supported by different subsystems, as in the dual-mechanism theory.

These studies have raised several concerns. One is whether they tested a hypothesis that would distinguish between the competing theories. It has been assumed that any evidence that regulars and irregulars differ supports the dual-mechanism theory (see, e.g., Beretta et al., 2003). However, connectionist models such as Joanisse and Seidenberg's (1999) do not predict that regulars and irregulars should behave alike in all respects. To the contrary, our model suggests that regulars and irregulars tend to differ with regard to the roles of phonological and semantic information, respectively. Thus, the issue is not whether there is a global difference between regulars and irregulars. Rather, we need to know two things: First, is the regular-versus-irregular distinction sufficient to account for observed patterns of brain activity? For example, there could be an overall difference between regulars and irregulars, but some irregulars nonetheless pattern with regulars. Second, what types of information underlie any observed differences between regular and irregular forms? One theory holds that they are processed by different types of mechanisms (e.g., a rule, an associative net); the other theory holds that they differ with respect to reliance on semantic versus phonological information. ${ }^{3}$ It should be possible to use imaging data to distinguish between these theories.

Previous neuroimaging studies of past tense have also raised methodological issues, as discussed by Seidenberg and Hoeffner (1998) with respect to the Jaeger et al. (1996) PET study and by Seidenberg and Arnoldussen (2003) concerning the Beretta et al. (2003) fMRI study. Many of the issues relate to design limitations imposed by a given neuroimaging technique. For example, the Jaeger et al. study used a blocked design such that regulars and irregulars were presented separately, which introduces problems because regular past tenses are more homogeneous in form (they all end with the past-tense morpheme spelled -ed) than irregular past tenses (which are formed in a variety of ways). This difference can promote conditionspecific effects based on the predictability or redundancy of the stimuli. The visual presentation of present tenses to elicit past tenses (Beretta et al., 2003; Dhond et al., 2003; Lavric et al., 2001) creates additional pitfalls concerning the effects of orthography or orthographic-phonological consistency. Visual presentation may also encourage participants to adopt a strategy in which they add -ed to the end of the target word and then judge whether it is a valid past tense (Dhond et al., 2003), which could again make processing regulars and irregulars artificially more different than is actually the case.

Methodological issues aside, these studies did not reveal the strong dissociations between the two types of forms that are predicted by the dual-mechanism account.
Such theories emphasize the modular, isolable character of the two subsystems and the fact that they are governed by different principles, which are thought to be represented in different brain regions. Both types of verbs should activate some of the same areas because of aspects of the past-tense generation task that are common across trials (e.g., recognizing a present-tense verb, planning and executing an articulatory output). However, the dissociable subsystems view suggests that some areas should be more strongly activated for rule-governed verbs and others more strongly activated for exceptions.

Neuroimaging studies have not yielded these dissociations. In the Jaeger et al. (1996) study, the past tenses of verbs (regulars, irregulars, and nonce verbs such as wug) were observed to have activated broad areas of cortex. As Seidenberg and Hoeffner (1998) noted, irregular verbs and nonwords, which are presumed by the dual-mechanism theory to be processed by separate subsystems, actually activated more areas in common than did regular verbs and nonwords, which are presumed to be processed by the same system. Beretta et al. (2003) found greater activation for irregulars in both frontal and posterior temporal regions, but no region showed greater activation for regular forms. This type of single dissociation is open to alternative interpretations, including the simple possibility that this pattern reflects differences in the difficulty of stimuli, such that stimuli requiring greater processing overall (the irregular forms) yielded greater degrees of activation than did easier stimuli (rule-governed ones) across multiple brain regions (Seidenberg \& Arnoldussen, 2003). Such a confound between the stimuli could be eliminated by selecting regular and irregular forms that are equally difficult to generate (e.g., stimuli that are matched for reaction times and error rates), but that was not the case in the Beretta et al. study.

Pinker and Ullman (2002) have suggested that the two mechanisms involved in past tense map onto discrete brain regions that correspond to areas that are affected in patients with selective past-tense deficits. They proposed that rule-based forms are processed in regions of left inferior frontal gyrus (L-IFG) and possibly subcortical regions that project onto L-IFG. Irregulars are processed in associative memory systems residing in the posterior portion of the left temporal lobe. Again, however, previous neuroimaging studies have not yielded results consistent with these predictions. Jaeger et al. (1996) found some regions that yielded dissociations between regulars and irregulars, but they did not map onto the predicted L-IFG and posterior temporal regions. Similarly, Beretta et al. (2003) found greater activation for irregulars than for regulars in IFG, which is where Pinker and Ullman (2002) place the regular mechanism.

Electrophysiological studies have also yielded dissociations between regulars and irregulars, but here again the results have been inconsistent. In a MEG study, Dhond et al. (2003) identified largely overlapping activity in temporal and frontal brain regions for regulars and ir- 
regulars. The authors found only small dissociations between these forms, with regulars evoking slightly more activation in L-IFG about $470 \mathrm{msec}$ post stimulus onset, and irregulars showing a similar advantage in left inferior temporal cortex at $340 \mathrm{msec}$. Lavric et al. (2001) used ERPs to identify differences between waveforms evoked by regulars and irregulars, but their results were appreciably different from those of Dhond et al., both with respect to estimated brain regions that showed the dissociation and the time points at which they occurred. Low-resolution electromagnetic tomographic analysis (LORETA) in the Lavric et al. study indicated that the sources of activity differences for regulars were located in right superior and medial frontal gyri and the right superior and middle temporal gyri, whereas irregulars were associated with activity in left superior, middle, and inferior temporal gyri and the anterior cingulate bilaterally; these differences occurred at 288-321 msec following stimulus onset. The disagreement between electrophysiology studies with respect to which regions show regular/ irregular dissociations, and the fact that they occurred only during very narrow time windows, again seems inconsistent with the theory that the brain makes a broad distinction between rule processing in L-IFG and recalling irregulars in left posterior temporal cortex. In summary, the evidence that emerged from initial attempts to bring neuroimaging and electrophysiological data to bear on the rules-versus-connectionist debate has not been decisive.

The rationale for the present study was to build on these earlier experiments, addressing some of the methodological issues that they raised (e.g., artifacts due to blocked presentation of conditions, difficulty confounds, and modality of stimulus presentation). However, our study differs from the earlier ones in a more basic way: We did not merely look for differences between regulars and irregulars. Rather, we also included stimuli and analyses that tested predictions derived from the connectionist theoretical framework. Dual-mechanism theories incorporate a strong distinction between rule-governed forms and exceptions: Every form is categorized as one or the other (modulo the existence of a small number of forms that are undergoing diachronic change and thus permit both forms, e.g., dreamed/dreamt). In contrast, connectionist models incorporate the idea that how well a word (e.g., a past-tense verb) is formed is a graded function of multiple probabilistic constraints. The main constraints derive from statistics about the mappings between codes (e.g., semantics and phonology) that are observed across a large number of lexical items. Words differ in the degree to which they satisfy these constraints. This yields a continuum ranging from highly consistent patterns (e.g., the standard present-past-tense transformation) to highly inconsistent patterns (e.g., suppletive verbs such as go-went); crucially, this account predicts intermediate cases exhibiting partial regularities. Of interest in the present study was whether these graded dis- tinctions between regulars and irregulars would be reflected in brain activity.

Participants were imaged in an event-related fMRI paradigm as they listened to present-tense verbs and covertly generated their past-tense forms. We examined patterns of brain activity for regular verbs (words and nonwords) and irregular past-tense verbs such as took. In addition, we included "pseudoregular" past-tense verbs such as slept that are irregular according to the dual-mechanism approach, but that are phonologically similar to regular verbs. As discussed below, the two theories make different predictions about these items. Nonce verbs were also included to assess generalization in the absence of prior word-specific information. This condition provides perhaps the most straightforward case of regular past-tense usage, given that past tenses of nonwords tend to be regular and must be generated by generalization from known forms. They cannot be recalled from memory the way familiar forms might be. In order to address whether any differences in brain activity were due to inadvertent differences between conditions with respect to item difficulty or familiarity, we also conducted a behavioral experiment in which adult English speakers performed the same elicitation task outside the magnet, yielding generation latency and accuracy data.

As in previous studies, we investigated whether activation in specific brain regions differentiates processing of regular and irregular forms. However, this comparison alone may not suffice to distinguish between the two theories. Dual-mechanism theories suggest that such a dissociation would reflect independent processing modules that are involved in processing rule-based and lexicalized forms. According to the connectionist theory, what matters is the extent to which a given word overlaps with other words in terms of constraints that affect processing. Thus, differences between regulars and irregulars could be due to phonological and semantic differences between these items (for a discussion, see Joanisse \& Seidenberg, 1999, and McClelland \& Patterson, 2002).

To obtain information that provided a stronger test of the two theories, we examined a further hypothesis - that irregulars should fractionate into two subtypes. We contrasted irregulars such as took, which overlap relatively little with regular past-tense forms, with pseudoregular irregulars such as slept, which are phonologically similar to regulars in several ways. The rule-based past tense of sleep would be sleeped, and so slept is standardly treated as an irregular. However, slept is not arbitrarily related to sleep; the irregularity in slept arises from the vowel change on the stem, not the inflection. Thus, it contains the $/ \mathrm{t} /$ ending that is correct for many past tenses, and it also ends in a consonant cluster, as do many regulars (e.g., stepped). In addition, the mapping between sleep and slept is partially consistent owing to its similarity to other irregular forms that involve a vowel change and the addition of [d] or [t] (weep-wept, keep-kept, tell-told, sell-sold). On the basis of these facts, we cate- 
gorize forms such as slept, heard, and meant as pseudoregulars rather than as true irregulars.

The connectionist theory predicts that pseudoregulars will tend to pattern with regular verbs with respect to the activation of brain regions associated with phonological processing - for example, areas of IFG. In contrast, irregular past tenses such as took overlap less with regular past tenses and so should produce less activation in phonological regions. The theory further predicts that no other regions will be found that dissociate regulars from both true irregulars and pseudoregulars. Together, these results would indicate that past-tense processing is not organized in terms of the regular-irregular distinction, but rather with respect to properties such as phonological similarity. In contrast, the dual-mechanism theory predicts that the brain activity associated with pseudoregular forms will be determined by the fact that they are irregular; hence, they should pattern with true irregulars (e.g., took and woke) and differ from rule-governed past tenses. Thus, the dual-mechanism theory predicts areas of activation that will dissociate regulars from both true irregulars and pseudoregulars; the connectionist theory predicts no such regions.

We expected to observe significant brain activation in the inferior frontal gyrus and the posterior portion of the temporal lobe. Given the results of earlier verb studies (Beretta et al., 2003; Dhond et al., 2003), it also seemed likely that these effects would be observed in both left and right hemispheres, although perhaps to a greater degree in the left hemisphere. Activation in IFG can be accounted for by the role that this region plays in phonological processing, and that activation in temporal regions would reflect semantic processing components of the past-tense generation task. These predictions draw in part on patient data indicating that injuries to these regions differentially affect semantic and phonological aspects of past tense. For instance, patients with Broca's aphasia tend to have difficulty with phonological processing (Bird, Lambon Ralph, Seidenberg, McClelland, \& Patterson, 2003; Tyler, Randall, \& Marslen-Wilson, 2002), whereas damage to inferior temporal lobe regions has been associated with semantic impairments (Patterson, Lambon Ralph, Hodges, \& McClelland, 2001). These results are consistent with the theory that past-tense generation normally depends on a conjunction of phonological and semantic constraints and that phonological and semantic deficits are the proximal cause of dissociations in regular and irregular morphology, such that patients with LIFG lesions have problems with nonword past tenses, whereas patients with temporal lobe lesions have greater difficulty with irregulars (Joanisse \& Seidenberg, 1999; McClelland \& Patterson, 2002).

\section{METHOD}

\section{Participants}

Imaging data were obtained from 10 right-handed participants ( 5 female, 5 male) in London, ON. The mean age of the participants was 26.8 years (range: $22-32$ years). Behavioral measures of past- tense naming were also obtained for the same stimuli from 24 participants who were not included in the imaging experiment. These participants were right- and left-handed monolingual Englishspeaking adults who were enrolled in undergraduate studies at the University of Western Ontario. The participants in both studies received either monetary compensation or course credit. All experimental procedures were approved by the University of Western Ontario Review Board for Health Sciences Research involving Human Subjects. All participants were neurologically healthy and reported normal hearing.

\section{Stimuli}

There were 60 stimulus items, consisting of 16 nonwords and 44 English present-tense verbs (16 regulars, 16 true irregulars, 12 pseudoregulars; see Appendix A). The three familiar word lists were equated listwise on log past-tense frequency (Zeno, Ivens, Millard, \& Duvvuri, 1995), imageability, and concreteness (Coltheart, $1981 ; p>.05$ for all comparisons). There were no log frequency differences between the pseudoregular, irregular, and regular verbs ( $p>.05$ for all three comparisons).

\section{Procedures}

The participants in the behavioral study heard each present-tense verb via headphones and were asked to say the past-tense form as quickly as possible. Response times were recorded using a voiceactivated response key. Responses were recorded to audiotape and scored later for accuracy. We also scored responses for types of errors, specifically overregularizations (e.g., breaked), stem distortions (take-talked), and no-change errors (stem repetition). Regularizations were considered the correct responses for nonwords.

Prior to scanning, the participants were given practice on the generation task, using six items (two regulars, two irregulars, and two nonwords) that were not included among the stimuli in the experiments. This provided practice on the past-tense generation task and helped to ensure that they understood the task. During scanning, the participants performed 60 trials divided equally across two separate runs acquired within a single session. Auditory stimuli consisted of digital waveforms (16-bit, 22200-Hz sample rate) spoken by an adult male native English speaker and presented using a custom fMRIcompatible sound system that conducted audio signals from a pair of piezoelectric speakers to a headset via PVC tubes. The headset also served to attenuate scanner noise during the experiment. The participants performed a past-tense verb generation task in which they heard a present-tense verb or nonword and were asked to think of its past tense. Overt spoken responses were not used because of the effect of movement artifacts in scanning.

MR scanning was performed using a 4-tesla Varian/Siemens scanner equipped with a hybrid quadrature head coil for signal transmission and reception. The participants' heads were stabilized using foam padding. Functional scans were acquired using a T2weighted, navigator echo-corrected, interleaved two-shot gradient EPI pulse sequence for blood oxygen level dependent (BOLD) imaging (Ogawa et al., 1992). Volumes consisted of eleven to twelve $64 \times 64$ transverse-oriented 5-mm slices with an in-plane resolution of $3 \times 3 \mathrm{~mm}$ (no interslice gap, volume acquisition time $=1.2 \mathrm{sec}$ [1.0 sec for participants 1 and 2], ${ }^{4} \mathrm{TE}=15 \mathrm{msec}$, field of view $=$ $192 \times 192 \mathrm{~mm}$, flip angle $=30^{\circ}$, bandwidth $=140.35 \mathrm{kHz}$ ). Ten fMRI volumes were acquired between trials in order to obtain eventrelated hemodynamic response curves for each participant on each condition. Functional volumes were oriented in such a way as to acquire the entire posterior temporal lobe, in addition to the middle and inferior frontal lobe, using a T1 sagittal scout image as reference. Scans also covered the entire occipital lobe, the anterior portion of the middle and superior temporal lobe, and the inferior portion of the parietal lobe. To compensate for magnetic saturation effects, the first three volumes of every scanning session were discarded from analysis. High-resolution anatomical scans were ob- 
tained within session and consisted of high-resolution, whole-brain $256 \times 256 \times 128$ T1-weighted scans using a 3-D segmented, driven equilibrium FLASH sequence $(\mathrm{FOV}=192 \times 192 \times 160 \mathrm{~mm}$, in-plane resolution $=0.8 \mathrm{~mm}$, slice thickness $=1.25 \mathrm{~mm}$, inversion time $=600 \mathrm{msec}, \mathrm{TE}=5.5 \mathrm{msec}, \mathrm{TR}=10.0 \mathrm{msec}$, flip angle $=$ $15^{\circ}$, bandwidth $=62.50 \mathrm{kHz}$ ).

\section{fMRI Data Analysis}

Imaging data were analyzed using the BrainVoyager analysis tools (Brain Innovation BV; Maastricht, The Netherlands). Functional scans were preprocessed using large-vessel suppression (Menon, 2002), 3-D rigid-body motion correction, Gaussian filtering in the temporal domain (full-width, half-maximum of 2 volumes) and spatial domain (full-width, half-maximum of 2 voxels in all dimensions), and mean intensity adjustment. Functional and anatomical scans were coregistered across participants by transforming them into the 3-D stereotaxic space of Talairach and Tournoux (1988). Groupwise functional maps were transformed into an inflated cortical surface template for data visualization purposes.

Statistical analyses were performed in 3-D Talairach space using a random-effects general linear model (GLM) with independent predictors for each stimulus type (regular, nonword, pseudoregular, and true irregular). Regions of significant activation for each condition were identified by comparing voxel time courses to a canonical BOLD response predictor function (gamma wave function; delta, 2.5; tau, 1.25; task duration, 1 volume). A random-effects analysis was used to identify significant voxels across participants exceeding a statistical threshold $[t(9)=4.8, p<.0002]$, corrected for multiple comparisons. The corrected $p$ value was obtained using AlphaSim, a 10,000-iteration Monte Carlo simulation of the acquisition volume $(64 \times 64 \times 12$ voxels $)$, taking into account a fullwidth half-maximum of 2 voxels spatial smoothing and an uncorrected voxelwise significance level $(p<.001)$, yielding a minimum cluster size of $135 \mathrm{~mm}^{3}$ (Forman et al., 1995; Xiong, Gao, Lancaster, \& Fox, 1995).

In the first analysis, a GLM contrast was used to identify whether specific brain regions differentiated all regular past tenses from all irregular past tenses. This was done by contrasting the combined regular and nonword conditions with the combined pseudoregular and true irregular conditions. A second GLM was used to identify regions of activation for the past-tense processing task as a whole. This was done by identifying voxels that reached significance across all four predictor functions. This second analysis allowed us to compare mean activation levels for each condition without assuming that independent brain regions were differentiating regular and irregular forms.

For both analyses, conditionwise activation levels were compared within significant clusters of voxels using each participant's average event-related BOLD responses for each condition, normalized to the mean signal level 1 volume before stimulus presentation. The estimated signal level was obtained using the average event-related BOLD signal level of each condition at 2-6 volumes post trial onset (to compensate for a shorter volume acquisition time in Participants 1 and 2, the average signal levels at 2-7 volumes were used for those participants). Means were calculated for all significant voxels within each anatomical region of interest. Repeated measures $t$ tests ( $\alpha=.05$, one-tailed) were used for planned comparisons of regulars and nonwords versus pseudoregulars, true irregulars, and the combined irregular lists.

\section{RESULTS}

\section{Behavioral Study}

Results of the behavioral study are presented in Table 1. A one-way analysis of variance (ANOVA) based on the mean generation latencies of the four types of verbs (reg-
Table 1

Reaction Times (RTs) and Accuracy Rates From the Behavioral Study

\begin{tabular}{|c|c|c|c|c|}
\hline \multirow[b]{2}{*}{ Type of Verb } & \multicolumn{2}{|c|}{ RT (msec) } & \multicolumn{2}{|c|}{ Accuracy } \\
\hline & $M$ & $S D$ & $M$ & $S D$ \\
\hline Regular & 746 & 339 & .94 & .07 \\
\hline Pseudoregular & 692 & 512 & .82 & .13 \\
\hline True irregular & 717 & 295 & .85 & .16 \\
\hline Nonword & 1,061 & 537 & .74 & .17 \\
\hline
\end{tabular}

ulars, pseudoregulars, true irregulars, and nonwords) was significant $[F(1,23)=15.76, p<.001]$. Post hoc tests indicated that latencies were similar among the three familiar word conditions, but all differed from the nonword condition ( $p<.001$ for all three comparisons). A second one-way ANOVA based on the percent correct responses in each of the four conditions was also significant $[F(1,23)=11.92, p<.001]$. This effect was obtained, as indicated by $t$ tests, because accuracy was higher for the regular verbs than for the other three conditions, which did not differ from one another $(p>.05$ for all three comparisons). We also observed that the percent overregularizations per item of the pseudoregulars $(M=$ 7.3; $S D=13.3$ ) was not significantly different from that of the true irregular items $[M=9.6 ; S D=15.0 ; F(1,26)=$ $2.17, p>.05]$.

The behavioral data indicate that participants were slower to generate past tenses for nonwords than for familiar verbs, which did not differ from each other. They also showed higher accuracy for regulars than for true irregulars, pseudoregulars, and nonwords. However, participants did not differ on true irregulars and pseudoregulars in terms of error rates, nor did they show a greater tendency to overregularize pseudoregular forms (e.g., producing weeped instead of wept). Since there was no indication that the two types of irregulars differed in difficulty, any fMRI signal differences between them cannot be attributed to factors related to response latency or accuracy.

\section{fMRI Study}

The first analysis was designed to identify regions of activation that differentiated regular and irregular formsthe most straightforward test of the dual-mechanism theory. In this analysis, regulars and nonwords were treated as one condition, and pseudoregulars and true irregulars were treated as a second condition. This grouping corresponded to the distinction between rule-governed forms and exceptions in the dual-mechanism theory. The two conditions were contrasted using a random-effects GLM. No significant cluster of activation was obtained for the combined irregulars $>$ word and nonword regulars contrast, suggesting that no brain regions in this experiment were significantly more activated when processing irregular forms. However, we did identify a cluster of voxels that was significant for the word and nonword regulars $>$ combined irregulars: an area of right IFG and insula $(x=$ $37, y=16, z=0$, BA 13; see Figure 2A). 


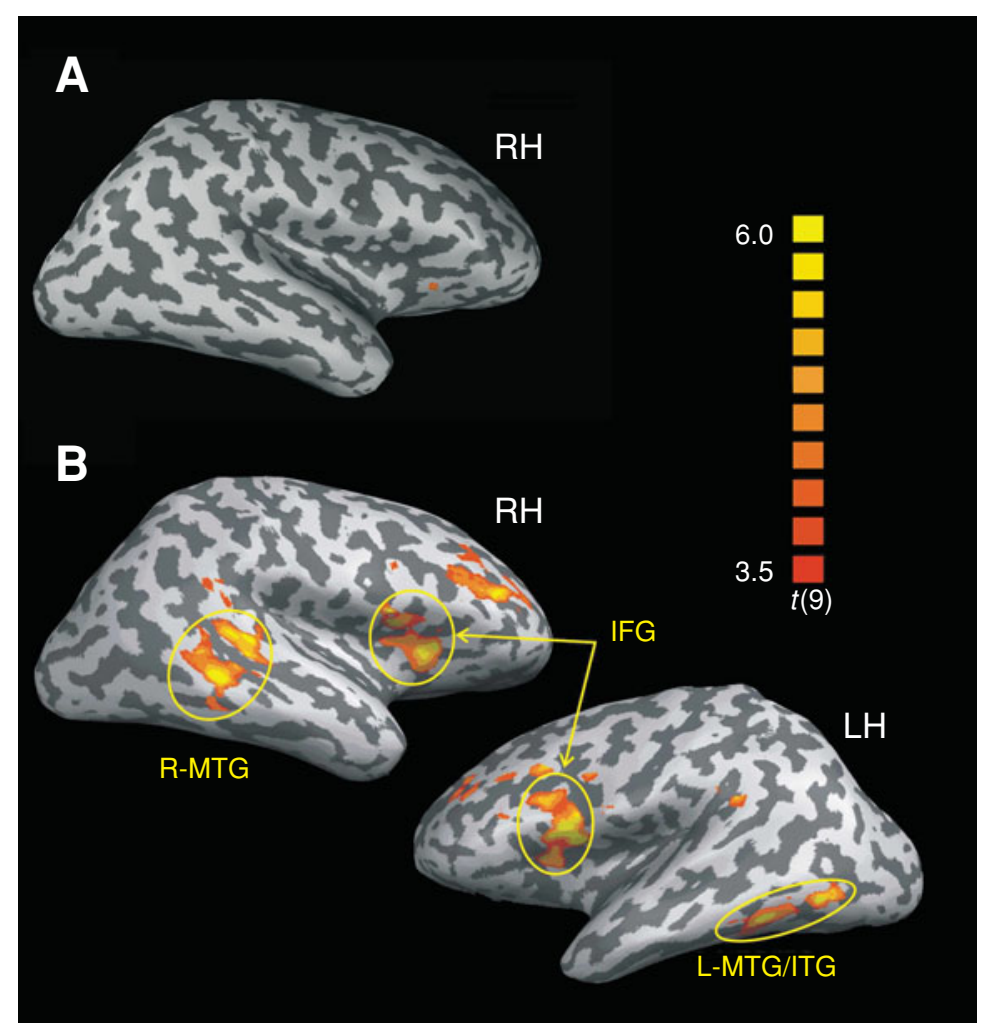

Figure 2. (A) A cluster of significant voxels in right inferior frontal gyrus (IFG) showing greater activation for the combined regular word and nonword conditions than for the combined irregular conditions. The statistical map has been overlaid onto an inflated 3-D rendering of the cortical sheet of one of the participants. (B) Regions of significant fMRI activation for the past-tense generation task as a whole, overlaid onto an inflated 3-D brain. Statistical maps were obtained using a random-effects general linear model, which identified significant voxels across all conditions. The circled clusters of significant voxels were used in subsequent analyses of conditionwise activation within each region.

Planned comparisons were next used to compare mean BOLD signal levels in this significant region for each of the four conditions in this experiment (Figure 3). To address the different predictions of the dual- and singlemechanism theories with respect to how pseudoregular forms compare with true irregulars, these two lists were first analyzed together as one condition ("combined irregulars") and then as separate conditions. Activation was greater for both the regular and the nonword conditions than for the combined irregulars (regular - combined irregular, $t=2.64, p<.05$; nonword - combined irregular, $t=2.16, p<.05)$. However, the advantage for regulars and nonwords was not preserved when the two were compared with pseudoregulars alone $(p>.05$, both comparisons). In contrast, the true irregular condition was significantly different from both the regular and the nonword conditions $(t=2.94, p<.01 ; t=2.54, p<.05$, respectively). These results are inconsistent with the dual-mechanism theory: One type of irregular ("true") was different from regulars but the other type ("pseudoregulars") was not. Thus, the relative activation in IFG reflects phonological factors rather than following the rule-versus-exception distinction.

We also considered whether the failure to identify regions that reliably differentiated all rule-governed forms from all exceptions was simply due to the inclusion of nonwords in the whole-brain analysis. An additional randomeffects GLM contrast was performed on the whole-brain data in which only the regular list was contrasted with the combined true irregular and pseudoregular conditions. This analysis did not reveal any significant regions of activation, which again suggests that regulars alone do not activate reliably different regions in comparison with the two types of irregulars.

We next employed a different approach to comparing activation across conditions: Using a random-effects GLM, we identified regions that were significantly activated when all conditions of the past-tense task were averaged together. This analysis revealed significant activation in several brain regions that are typically observed during auditory word processing (Figure 2B; sizes and coordinates of significant regions are listed in Appendix B). 


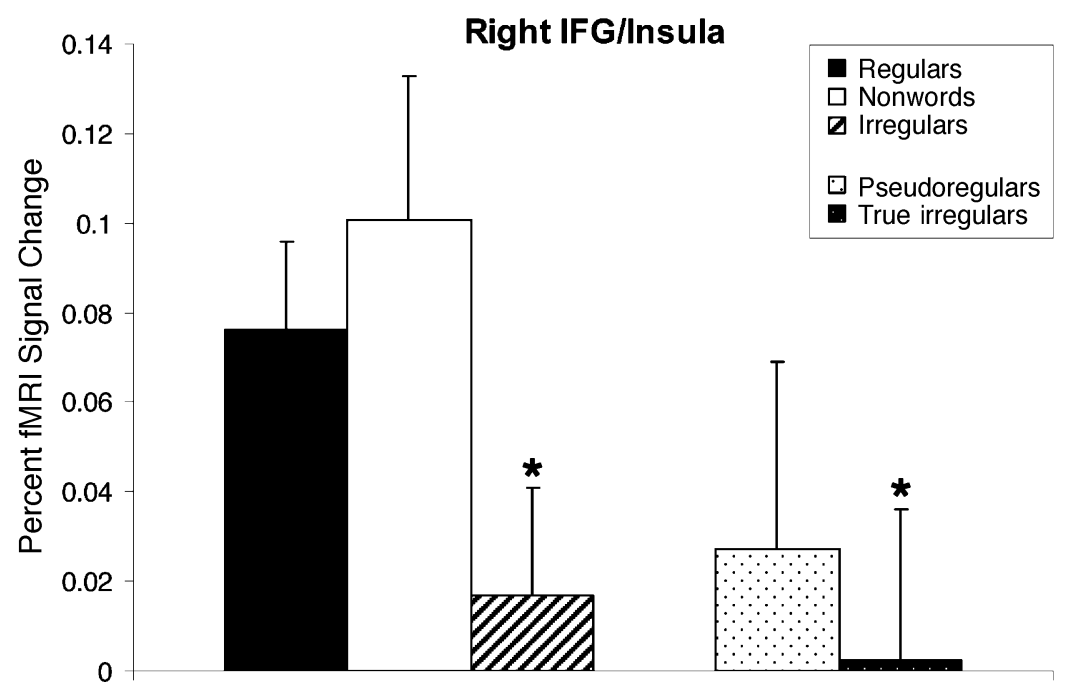

Figure 3. Comparison of mean conditionwise activation levels (percent fMRI signal change) within activated regions of right IFG and insula. *Significantly different from regulars and nonwords, $p<.05$.

These included posterior areas of the left and right temporal lobes extending across the superior, middle, and inferior temporal gyri (STG, MTG, and ITG), as well as the left and right IFG. We also observed activation in the right middle frontal gyrus (R-MFG).

We then examined whether the overall activation levels in any of these regions were being carried by different types of verbs. This was done using planned comparisons of mean conditionwise signal levels for each of the four conditions, as was done for the rule-versus-exception contrast above. We first examined activation levels in frontal regions (Figure 4). Regulars showed activation levels significantly greater than those for the combined irregulars in L-IFG $(t=1.87, p<.05)$ and in R-IFG $(t=1.92$, $p<.05)$. We also observed a similar finding for nonwords in comparison with the combined irregulars (L-IFG: $t=1.93, p<.05$; R-IFG: $t=2.90, p<.01)$. No differences between conditions were observed in the R-MFG region of activation. At first glance, these results suggest a region of IFG specialized for regular forms, which was too subtle to be captured by the whole-brain GLM contrast performed earlier. However, a key question in this experiment was whether such an effect was due to differences in how this region processed regular forms, or whether it was instead influenced by phonological properties of the verbs. We tested this by comparing activation for the pseudoregular and true irregular conditions separately with the nonword and regular conditions. If the advantage for regulars in IFG was due to the morphological properties of these words, both types of irregulars should contrast equally well with the regular and nonword conditions. However, if activation differences reflected phonological properties of words, as the connectionist theory predicted, we instead expected that activation for pseudoregulars would tend to be similar to that for regulars and nonwords, whereas activation for the true irregulars would tend to be weaker than that for regulars and nonwords.

Mean fMRI signal levels in L-IFG were greater for both the regular and nonword conditions than for the true irregular condition (regulars vs. true irregulars, $t=2.21$, $p<.05$; nonwords vs. true irregulars, $t=2.29, p<.05$ ). However, consistent with the predictions of the connectionist theory, the pseudoregulars did not differ from either the regular or nonword conditions $(p>.05$, both conditions). Moreover, an additional $t$ test revealed that activation was significantly greater for the pseudoregulars than for the true irregulars $(t=2.17, p<.05)$. Similar results were observed in the R-IFG region of activation, marked by significantly greater signal levels for both the regular and nonword conditions than for true irregulars $(t=2.94, p<.01$, and $t=2.54, p<.05$, respectively), but not for the regulars or nonwords in comparison with the pseudoregulars ( $p>.05$, both conditions). These results suggest that differences in signal levels for regulars and irregulars in IFG in fact reflect a graded distinction between forms; that is, even though significant differences might occur between regular and irregular forms, these effects are likely due to factors related to phonological similarity rather than to the existence of a rule mechanism.

We also compared signal levels in regions of significant activation of right and left MTG and ITG (Figure 5). This analysis did not reveal any significant differences among conditions, both when regulars and nonwords were compared separately with the combined irregulars, and when true irregulars and pseudoregulars were separately compared with regulars and nonwords. The results suggest that verb regularity did not significantly modulate activation levels in this region. Thus, they indicate that 

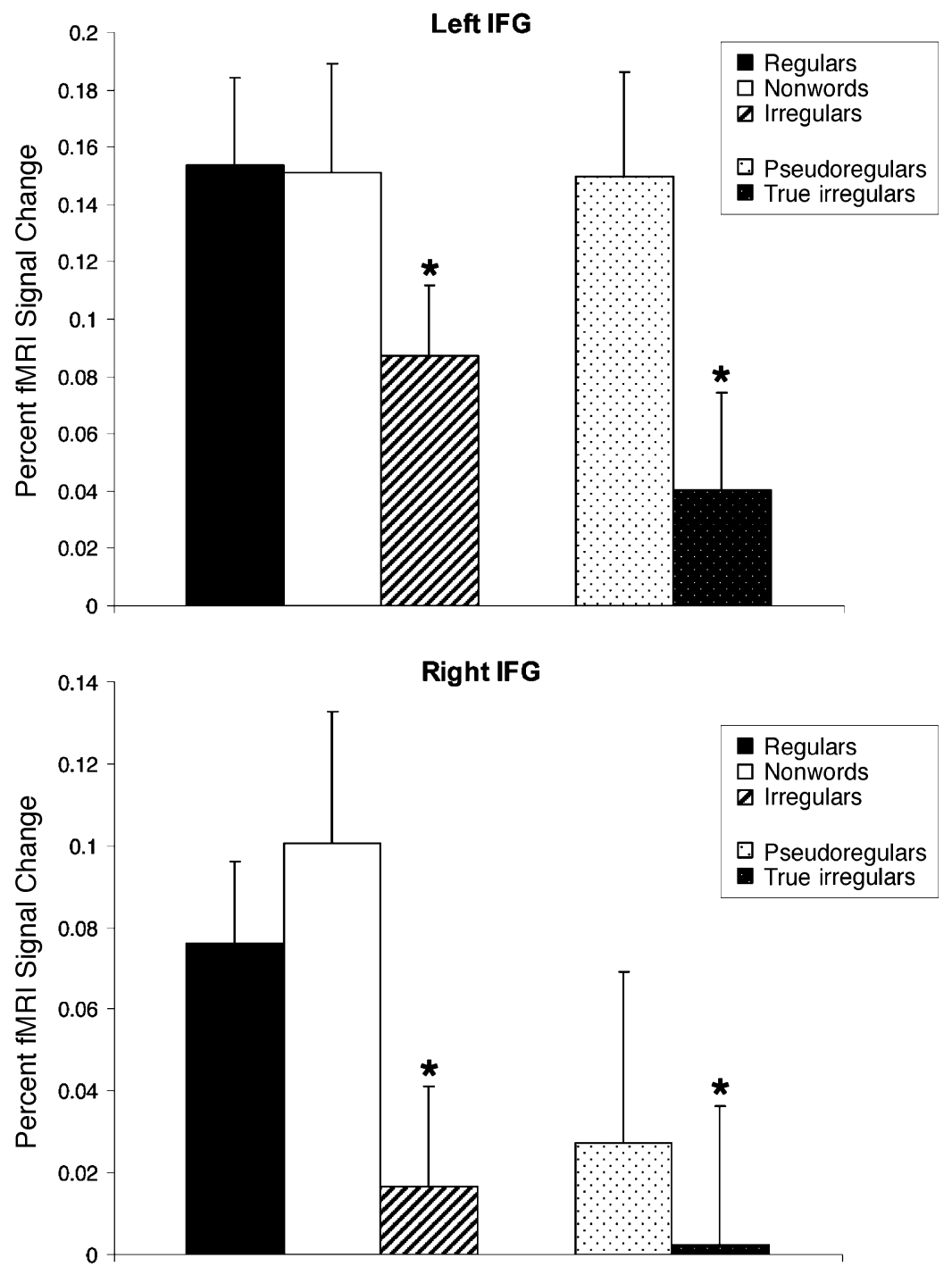

Figure 4. Comparison of mean conditionwise activation levels in activated regions of left and right IFG. The data illustrate an advantage for regulars and nonwords in this region, compared with the combined irregulars. However, pseudoregulars (i.e., irregulars that are phonologically similar to regulars, such as sleep-slept), evoked activation that was much closer to that for regular words and nonwords, in comparison with true irregulars (take-took). *Significantly different from regulars and nonwords, $p<.05$.

the advantage for word and nonword regulars over true irregulars was isolated to frontal regions, rather than being a broad effect that occurred across all activated regions.

\section{DISCUSSION}

In the present study, we sought to provide novel neuroimaging evidence bearing on the controversy concerning rule-based and connectionist approaches to language. The critical question was whether the regular-irregular dichotomy that is central to the dual-mechanism theory would correctly predict patterns of brain activity. We contrasted this hypothesis with the prediction, derived from the connectionist account, that regular-like irregu- lars (which we have termed pseudoregulars) will behave more like regulars than like other irregulars. In the behavioral experiment, generation latencies tended to be longer for nonwords than for familiar verbs, and accuracy was higher for regulars than for nonwords, true irregulars, and pseudoregulars. Critically however, the true irregular and pseudoregular conditions did not differ on either behavioral measure, nor did they differ with respect to overregularization rates. Thus, fMRI differences between the true irregulars and pseudoregulars did not occur because the latter were harder to generate or had a stronger tendency to be regularized.

We next used fMRI to identify regions of brain activation implicated in past-tense processing. The first analy- 

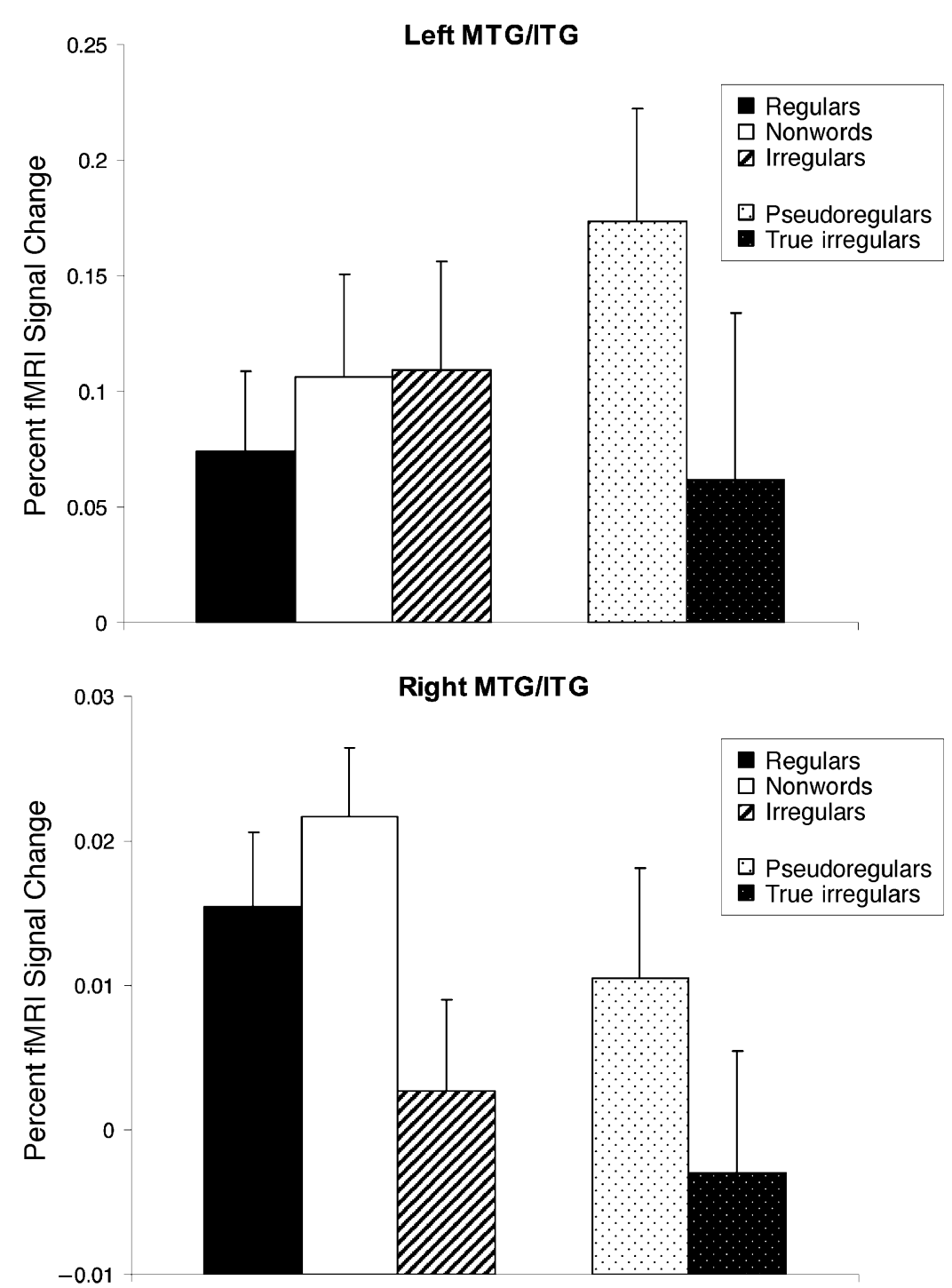

Figure 5. Mean conditionwise BOLD signal levels for each condition within activated regions of left and right MTG and ITG. No significant differences in activation across conditions were observed, suggesting that these regions were not differentiating past-tense subtypes in this experiment.

sis focused on identifying regions that dissociated putatively rule-governed forms (word and nonword regulars) from exceptions (the combined true irregulars and pseudoregulars). This failed to reveal any region showing greater activation for the combined irregulars than for rule-like forms; however, one cortical region in R-IFG showed more activation for word and nonword regulars than for the combined irregulars. This result could be construed as supporting the dual-mechanism theory, which holds that some regions of IFG are specifically involved in processing morphological rules but not in processing exceptions to these rules (see, e.g., Ullman, 2001). However, pseudoregulars patterned with word and nonword regulars in inferior frontal regions, with all three conditions producing similar levels of activation, all of which differed from the true irregulars. This result is inconsis- tent with a dual-mechanism theory (because the pseudoregulars should have patterned with other exceptions) but consistent with the connectionist theory (because the phonological similarity and regularity of regular and pseudoregular past tenses predicts that they should pattern together).

The failure to find cortical regions that reliably dissociate all regulars from exceptions nevertheless raises the possibility that processing different types of past tenses involves similar brain regions, but to systematically varying degrees. Such effects might have been too subtle to be captured by a contrast analysis of whole-brain data. To address this, in a second analysis we identified areas of significant activation across all conditions in the experiment and then compared mean conditionwise fMRI signal levels within each resulting region of interest. 
This analysis identified large areas of significant activation in the left and right hemispheres that included the posterior portion of the MTG, ITG, and IFG. We identified regions of IFG in both hemispheres that showed an advantage for word and nonword regulars compared with the combined irregulars. Here again, however, we found that activation for pseudoregulars was similar to that of both word and nonword regulars, rather than to that of the true irregulars. We also failed to observe any region that showed significantly different activation for both true irregulars and pseudoregulars, in comparison with regulars.

The explanation for these results seems to be that differences between regular past tenses and exceptions derive in part from phonological factors such as a form's relative phonological complexity, and also the consistency of the mapping between a present- and a past-tense form. Thus, activation was greater for regulars than for true irregulars because of the greater involvement of phonological mechanisms in processing regular forms. However, as predicted, this difference did not occur when regulars were compared with pseudoregular items, which consisted of pseudoregular items, "irregular" past tenses that are phonologically similar to regulars in two ways: They tend to have word endings that are phonologically more complex than those of other irregulars, and they involve partially regular mappings between present- and past-tense forms.

The analysis of regions activated across conditions also identified significant activation in bilateral MTG/ ITG. We have suggested that this activation corresponded to the semantic demands of the past-tense task; consistent with this, previous neuroimaging studies have also found activation in the same regions during semantic processing of words (see, e.g., Moore \& Price, 1999). We did not observe significant differences between verb conditions in these regions. We suggest that this is because in the past-tense generation task, there is activation of semantic information in all conditions. The participant is given a stimulus (typically a word, sometimes a nonword) and asked to generate its past tense. Listeners automatically access the meaning of the stimulus if it has one; in the case of nonwords, they compute information related to the concept of "pastness"; in some cases, nonwords also activate semantic information (Harm \& Seidenberg, 2004). Thus, semantic information is activated by all types of items in this task.

The findings of this experiment are consistent with the connectionist theory, which holds that processing both regular and irregular past tenses involves an interactive network of brain regions supporting phonological and semantic information. However, they are inconsistent with the dual-mechanism theory in three respects: (1) This theory predicts that frontal regions are minimally involved in processing all irregular forms; (2) it predicts that partial regularities are handled by lexical mechanisms responsible for exceptions, and therefore forms like slept should always pattern with other irregulars; and (3) it holds that the application of a morphological rule such as the past tense is independent of phonological factors, a point that Pinker has stressed repeatedly (Pinker, 1991, 1999; Pinker \& Ullman, 2002).

\section{Relationship to Aphasia}

The results of this imaging experiment complement recent work on past-tense production in aphasic patients. We have previously demonstrated in a neural network that lesioning phonological representations tends to affect the ability to generalize regular past tenses to nonwords, and that similar damage to semantic representations leads to difficulty with irregular verbs (Joanisse \& Seidenberg, 1999). These effects appear to correspond with what is observed in patients with Broca's aphasia in the case of phonological impairments, and Alzheimer's and semantic dementias in the case of semantic impairments (Bird et al., 2003; Patterson et al., 2001; Ullman et al., 1997).

With respect to irregulars, the model predicts that this type of information is critical to processing irregular past tenses, particularly lower frequency irregulars. This is because irregular verbs by definition have idiosyncratic past tenses, and, as a result, posterior brain regions implicated in semantic processing should play a stronger role for irregulars than for regulars. By the same token, the present results underline a critical distinction between how a specific brain region is implicated in normal processing and how actually lesioning this same region influences processing. The MTG/ITG area identified in this study is seen as necessary but not sufficient for processing all types of verbs, due to its role in retrieving words' semantic forms and in processing the concept of pastness. One consequence of this is that damage to inferior temporal regions has a greater impact on irregulars than on other types of past tenses, since past tenses taking regular endings can be generated without retrieving idiosyncratic information such as their meaning (Patterson et al., 2001). However, the single-mechanism theory also predicts that this region is equally important for generating past tenses of all verbs under normal circumstances; all words evoke semantic activation as listeners process them. As such, activation in this region would differ for regular and irregular past tenses as long as lexical/semantic variables were adequately controlled for.

The connectionist theory makes a different prediction about the role of inferior frontal regions and past tense: It predicts that patients with damage to such regions will tend to have difficulty with nonword past tenses because posterior regions cannot support unfamiliar formssimply put, nonwords by definition do not have a semantic form. In contrast, such a lesion will have less effect on both familiar verb types, since both regulars and irregulars can rely on semantic knowledge that supports generating their past tenses. That said, all verbs do tap some amount of phonological knowledge under normal circumstances, and as a result in the present study we observed similar degrees of activation in IFG for regulars and pseudoregulars, since these items had similar phonological characteristics. In the case of patients, Bird et al. 
(2003) observed that Broca's aphasics who have difficulty producing regular, as opposed to irregular, past tenses failed to show this effect when phonological complexity was controlled for. This result underlines the sensitivity of IFG regions to even relatively small differences in the phonological forms of words. It also supports the assertion that the critical variable in differences between regulars and irregulars with respect to inferior frontal regions is phonology, rather than morphology.

\section{Models of Frontal \\ and Temporal Activation in Language}

The present results are consistent with the hypothesis that activation in IFG and the inferior temporal lobe support phonological and semantic mechanisms known to play a role in morphology (Devlin, Jamison, Matthews, \& Gonnerman, 2004; Joanisse \& Seidenberg, 1999; Patterson et al., 2001; Seidenberg \& Gonnerman, 2000; Seidenberg \& Joanisse, 2003). This explanation also fits well with neuropsychological models of language in the left hemisphere that assume that regions of the posterior temporal lobe are involved in semantic processing and lexical retrieval (Martin, Wiggs, Ungerleider, \& Haxby, 1996; Price, Moore, Humphreys, \& Wise, 1997), whereas inferior frontal regions are responsible for speech production and phonological processing (Blumstein et al., 2000; Burton, 2001).

One complication to such an account, however, is that it is becoming increasingly clear that the anterior/posterior distinction between phonology and semantics is an oversimplification. In addition to semantic processing, regions of superior and middle temporal gyri have been found to play a role in phonological processing, especially as it relates to perception (Binder et al., 1997; Hickok \& Poeppel, 2000). Likewise, a growing literature implicates specific subregions of IFG in semantic retrieval (Devlin, Matthews, \& Rushworth, 2003; Gold \& Buckner, 2002; Poldrack et al., 1999). Nevertheless, the increasingly fine-grained model of functional specialization of language in these regions does not invalidate the general theory that we are putting forward here, that past-tense processing is subserved by multiple subregions of temporal and frontal cortex that are specialized for processing semantic and phonological information, and that distinct subregions are responsible for these capacities. Future studies may provide finer grained evidence as to the localization of regions subserving pasttense processing abilities, however.

\section{Conclusion}

The present results add to a growing body of evidence from computational modeling (Joanisse \& Seidenberg, 1999) and studies of brain-injured patients (Bird et al., 2003; Patterson et al., 2001) supporting the theory that past-tense morphology is processed as part of a distributed lexical network that encodes semantic, phonological, orthographic, and other types of word-related information. The pattern of activation that was observed in this study is better explained by the division of labor between these types of information in processing well-formed utterances than by the distinction between rule-governed forms and exceptions. Thus, the results support the theory that the key building blocks of language are sound and meaning, rather than "words and rules" (Pinker, 1999).

\section{REFERENCES}

Beretta, A., Campbell, C., Carr, T. H., Huang, J., Schmitt, L. M., Christianson, K., \& CaO, Y. (2003). An ER-fMRI investigation of morphological inflection in German reveals that the brain makes a distinction between regular and irregular forms. Brain \& Language, 85, 67-92.

Berko, J. (1958). The child's learning of English morphology. Word, 14, 150-177.

Binder, J. R., Frost, J. A., Hammeke, T. A., Cox, R. W., Rao, S. M., \& Prieto, T. (1997). Human brain language areas identified by functional magnetic resonance imaging. Journal of Neuroscience, 17, 353-362.

Bird, H., Lambon Ralph, M. A., Seidenberg, M. S., McClelland, J. L., \& PAtterson, K. (2003). Deficits in phonology and past tense morphology: What is the connection? Journal of Memory \& Language, 48, 502-526.

Blumstein, S. E., Milberg, W., Brown, T., Hutchinson, A., KurowSKI, K., \& BURTON, M. W. (2000). The mapping from sound structure to the lexicon in aphasia: Evidence from rhyme and repetition priming. Brain \& Language, 72, 75-99.

Burton, M. W. (2001). The role of inferior frontal cortex in phonological processing. Cognitive Science, 25, 695-709.

Bybee, J. L., \& Slobin, D. I. (1982). Rules and schemas in the development and use of the English past tense. Language, 58, 265-289.

Coltheart, M. (1981). The MRC psycholinguistic database. Quarterly Journal of Experimental Psychology, 33A, 497-505.

Daugherty, K., \& Seidenberg, M. S. (1992). Rules or connections? The past tense revisited. In Proceedings of the Fourteenth Annual Conference of the Cognitive Science Society (pp. 259-264). Hillsdale, NJ: Erlbaum.

Devlin, J. T., Jamison, H. L., Matthews, P. M., \& Gonnerman, L. M. (2004). Morphology and the internal structure of words. Proceedings of the National Academy of Sciences, 101, 14984-14988.

Devlin, J. T., Matthews, P. M., \& Rushworth, M. F. S. (2003). Semantic processing in the left inferior prefrontal cortex: A combined functional magnetic resonance imaging and transcranial magnetic stimulation study. Journal of Cognitive Neuroscience, 15, 71-84.

Dhond, R. P., Marinkovic, K., Dale, A. M., Witzel, T., \& Halgren, E. (2003). Spatiotemporal maps of past-tense verb inflection. NeuroImage, 19, 91-100.

Forman, S. D., Cohen, J. D., Fitzgerald, M., Eddy, W. F., Mintun, M. A., \& NolL, D. C. (1995). Improved assessment of significant activation in functional magnetic resonance imaging (fMRI): Use of a cluster-size threshold. Magnetic Resonance in Medicine, 33, 636-647.

Gold, B. T., \& Buckner, R. L. (2002). Common prefrontal regions coactivate with dissociable posterior regions during controlled semantic and phonological tasks. Neuron, 35, 803-812.

Halle, M., \& Mohanan, K. P. (1985). Segmental phonology of modern English. Linguistic Inquiry, 16, 57-116.

Harm, M. W., \& SeIdenberg, M. S. (2004). Computing the meanings of words in reading: Cooperative division of labor between visual and phonological processes. Psychological Review, 111, 662-720.

Haskell, T. R., MacDonald, M. C., \& SeidenberG, M. S. (2003). Language learning and innateness: Some implications of compounds research. Cognitive Psychology, 47, 119-163.

HickoK, G., \& PoepPEL, D. (2000). Towards a functional neuroanatomy of speech perception. Trends in Cognitive Sciences, 4, 131-138.

Jaeger, J. J., Lockwood, A. H., Kemmerer, D. L., Van Valin, R. D., Murphy, B. W., \& KhalaK, H. G. (1996). A positron emission tomographic study of regular and irregular verb morphology in English. Language, 72, 451-497.

JoAnisse, M. F., \& SeidenberG, M. S. (1999). Impairments in verb morphology after brain injury: A connectionist model. Proceedings of the National Academy of Sciences, 96, 7592-7597. 
Lavric, A., Pizzagalli, D., Forstmeier, S., \& Rippon, G. (2001). A double-dissociation of English past-tense production revealed by event-related potentials and low-resolution electromagnetic tomography (LORETA). Clinical Neurophysiology, 112, 1833-1849.

MacWhinney, B., \& Leinbach, J. (1991). Implementations are not conceptualizations: Revising the verb learning model. Cognition, 29, 121-157.

Marcus, G. F., Brinkmann, U., Clahsen, H., Wiese, R., \& Pinker, S. (1995). German inflection: The exception that proves the rule. Cognitive Psychology, 29, 189-256.

Marcus, G. F., Pinker, S., Ullman, M. [T]., Hollander, M., RoSEN, T. J., \& XU, F. (1992). Overregularization in language acquisition. Monographs of the Society for Research in Child Development, 57, 1-182.

Martin, A., Wiggs, C. L., Ungerleider, L. G., \& HaXby, J. V. (1996). Neural correlates of category-specific knowledge. Nature, 379, 649-652.

McClelland, J. L., \& PAtTerson, K. (2002). Rules or connections in past-tense inflections: What does the evidence rule out? Trends in Cognitive Sciences, 6, 465-472.

MENON, R. S. (2002). Postacquisition suppression of large-vessel BOLD signals in high-resolution fMRI. Magnetic Resonance in Medicine, $47,1-9$

Moore, C. J., \& PrICE, C. J. (1999). Three distinct regions for word and picture naming in the ventral visual pathway. NeuroImage, 10, 181-192.

Ogawa, S., Tank, D. W., Menon, R., Ellermann, J. M., Kim, S. G., Merkle, H., \& Ugurbil, K. (1992). Intrinsic signal changes accompanying sensory stimulation: Functional brain mapping with magnetic resonance imaging. Proceedings of the National Academy of Sciences, 89, 5951-5955.

Patterson, K., Lambon Ralph, M. A., Hodges, J. R., \& McClelLAND, J. L. (2001). Deficits in irregular past-tense verb morphology associated with degraded semantic knowledge. Neuropsychologia, 39, 709-724

Pinker, S. (1991). Rules of language. Science, 253, 530-535.

PINKER, S. (1999). Words and rules. New York: Basic Books.

Pinker, S., \& Prince, A. (1988). On language and connectionism: Analysis of a parallel distributed processing model of language acquisition. Cognition, 28, 73-193.

Pinker, S., \& Ullman, M. T. (2002). The past and future of the past tense. Trends in Cognitive Sciences, 6, 456-463.

Plunkett, K., \& Marchman, V. (1993). From rote learning to system building: Acquiring verb morphology in children and connectionist nets. Cognition, 48, 21-69.

Poldrack, R. A., Wagner, A. D., Prull, M. W., Desmond, J. E., Glover, G. H., \& Gabrieli, J. D. E. (1999). Functional specialization for semantic and phonological processing in the left inferior prefrontal cortex. Neurolmage, 10, 15-35.

Prasada, S., \& PinKer, S. (1993). Generalisation of regular and irregular morphological patterns. Language \& Cognitive Processes, 8, 1-56.

Price, C. J., Moore, C. J., Humphreys, G. W., \& Wise, R. J. S. (1997). Segregating semantic from phonological processes during reading. Journal of Cognitive Neuroscience, 9, 727-733.

RAMSCAR, M. (2002). The role of meaning in inflection: Why the past tense does not require a rule. Cognitive Psychology, 45, 45-94.

Rumelhart, D. E., \& McClelland, J. L. (1986). On learning the past tenses of English verbs. In D. E. Rumelhart, J. L. McClelland, \& the PDP Research Group (Eds.), Parallel distributed processing: Explorations in the microstructure of cognition. Vol. 2: Psychological and biological models (pp. 216-271). Cambridge, MA: MIT Press.

Seidenderg, M. S., \& Arnoldussen, A. (2003). The brain makes a distinction between hard and easy stimuli: Comments on Beretta et al. Brain \& Language, $\mathbf{8 5}, 527-530$

Seidenberg, M. S., \& Gonnerman, L. (2000). Explaining derivational morphology as the convergence of codes. Trends in Cognitive Sciences, 4, 353-361.

Seidenberg, M. S., \& Hoeffner, J. H. (1998). Evaluating behavioral and neuroimaging data on past tense processing. Language, 74, 104122

Seidenberg, M. S., \& Joanisse, M. F. (2003). Show us the model. Trends in Cognitive Sciences, 7, 106-107.

SPEnCER, A. (1991). Morphological theory. Cambridge, MA: Blackwell. TAlairach, J., \& Tournoux, P. (1988). Co-planar stereotaxic atlas of the human brain (M. Rayport, Trans.). New York: Thieme.

Tyler, L. K., Randall, B., \& Marslen-Wilson, W. D. (2002). Phonology and neuropsychology of the English past tense. Neuropsychologia, 40, 1154-1166.

Ullman, M. T. (2001). A neurocognitive perspective on language: The declarative procedural model. Nature Reviews Neuroscience, 2, 717 726

Ullman, M. T., Corkin, S., Coppola, M., Hickok, G., Growdon, J. H., KoroshetZ, W. J., \& Pinker, S. (1997). A neural dissociation within language: Evidence that the mental dictionary is part of declarative memory, and that grammatical rules are processed by the procedural system. Journal of Cognitive Neuroscience, 9, 266-276.

Xiong, J., Gao, J., Lancaster, J. L., \& Fox, P. T. (1995). Clustered pixels analysis for functional MRI activation studies in the human brain. Human Brain Mapping, 3, 1-15.

Zeno, S. M., Ivens, S. H., Millard, R. T., \& Duvvuri, R. (1995). The educator's word frequency guide. Brewster, NY: Touchstone Applied Science Associates.

\section{NOTES}

1. Whether the Joanisse and Seidenberg (1999) model entails a single mechanism, and therefore differs from the dual-mechanism alternative, has been questioned by Pinker and Ullman (2002), who note that our account involves the activation of multiple types of information (most prominently, semantic and phonological). The mechanism is a "single" one in the sense that exactly the same units and connections in the network are used in processing all forms. Different components of the model perform different functions, but these cannot be equated with the mechanisms in the dual-mechanism approach. The phonological units in our model encode the phonological forms of words, not a rule for generating the past tense. The semantic units encode the meanings of words; they do not implement a lexicon in the traditional sense of lexical entries specifying a word's spelling, sound, meaning, grammatical category, and so on. For simplicity, the Joanisse and Seidenberg model uses individual nodes to represent the semantics of individual words, but nothing about the results of our implementation turns on this choice; see Harm and Seidenberg (2004) for a similar model that computes past tenses using a distributed representation of semantics. Nor do such models differentiate into subsystems corresponding to those in the dual-mechanism theory; they do not allocate some units to processing exceptions and others to regulars. The semantic and phonological components of the Joanisse and Seidenberg model act conjunctively and simultaneously, not independently and serially. The same types of knowledge representation are used to represent all forms, and all the constraints are learned via the same mechanism.

2. Although Beretta et al. (2003) compared regular and irregular German nouns and verbs, the authors suggested that their results also apply to the past tense in English.

3. It is also worth noting here that we do not in fact predict that all regulars should differ from all irregulars. For instance, we predict that, when equated on critical factors such as phonological complexity, semantics (e.g., concreteness, animacy), and frequency, regulars and irregulars should behave similarly (Patterson, Lambon Ralph, Hodges, \& McClelland, 2001)

4. A hardware upgrade made it necessary to change the volume acquisition time parameter after the first 2 participants were run. We have adjusted for this by using a different event-related hemodynamic predictor function for the first 2 participants and modified the time points that were used to calculate their conditionwise average signal levels. 
APPENDIX A

Target Stimuli Used in the Past-Tense Elicitation Experiment

\begin{tabular}{|c|c|c|c|c|c|c|}
\hline \multirow[b]{2}{*}{ Regulars } & \multirow[b]{2}{*}{ Frequencies } & \multirow[b]{2}{*}{ Nonwords } & \multicolumn{4}{|c|}{ Combined Irregulars } \\
\hline & & & True Irregulars & Frequencies & Pseudoregulars & Frequencies \\
\hline tuned & 27 & murned & stole & 118 & leapt & 20 \\
\hline warmed & 125 & plagged & tore & 140 & dealt & 79 \\
\hline marched & 204 & pashed & sprang & 177 & fled & 165 \\
\hline tested & 213 & tassed & swam & 209 & slept & 403 \\
\hline raced & 247 & narted & slid & 235 & sold & 962 \\
\hline lined & 287 & zaned & chose & 351 & meant & 1,262 \\
\hline printed & 485 & zilled & sang & 421 & lost & 2,557 \\
\hline yelled & 671 & nalked & fought & 508 & felt & 4,213 \\
\hline waited & 921 & daced & drew & 777 & heard & 4,270 \\
\hline talked & 1,074 & smitted & met & 1,524 & told & 5,894 \\
\hline played & 1,232 & bleemed & fell & 1,819 & thought & 9,006 \\
\hline showed & 1,295 & froozed & sent & 2,299 & said & 47,719 \\
\hline passed & 2,241 & finted & built & 2,570 & & \\
\hline needed & 3,294 & tupped & stood & 2,826 & & \\
\hline wanted & 5,370 & suffed & held & 2,908 & & \\
\hline looked & 9,438 & woned & ground & 3,504 & & \\
\hline
\end{tabular}

Note-Frequencies of the past-tense forms are taken from Zeno et al. (1995).

\section{APPENDIX B}

Results of an Omnibus General Linear Model for the Verb Generation Task Indicating the Location and Extent of Clusters of Voxels Significant Across All Conditions

\begin{tabular}{|c|c|c|c|c|c|c|}
\hline \multirow[b]{2}{*}{ Region } & \multicolumn{3}{|c|}{ Talairach Coordinates } & \multirow{2}{*}{$\begin{array}{l}\text { Cluster Size } \\
\text { (No. Voxels) }\end{array}$} & \multicolumn{2}{|c|}{$\begin{array}{c}\text { Average } \\
\text { Statistical Value }\end{array}$} \\
\hline & $x$ & $y$ & $z$ & & $t$ & $p$ \\
\hline L-IFG/Insula (BA 13)* & -46 & 10 & 12 & 1,726 & 6.44 & .0003 \\
\hline L-MTG/ITG (BA 37)* & -52 & -57 & -7 & 546 & 5.55 & .0004 \\
\hline L thalamus & -13 & -2 & 14 & 195 & 5.50 & .0005 \\
\hline R-STG/MTG (BA 22)* & 54 & -34 & 4 & 744 & 5.44 & .0009 \\
\hline R-MFG (BA 9)* & 32 & 34 & 23 & 522 & 6.16 & .0003 \\
\hline R-IFG (BA 13)* & 29 & 22 & 8 & 1,271 & 5.99 & .0003 \\
\hline $\mathrm{R}$ thalamus & 11 & -4 & 12 & 2,139 & 5.51 & .0005 \\
\hline
\end{tabular}

Note-Statistical threshold, $t=4.8$; spatial threshold, 135 contiguous $1-\mathrm{mm}^{3}$ voxels above threshold. Asterisks indicate regions included in subsequent analyses. IFG, inferior frontal gyrus; MFG, middle frontal gyrus; STG, superior temporal gyrus; MTG, middle temporal gyrus; ITG, inferior temporal gyrus. 